\title{
List-Distinguishing Colorings of Graphs
}

\author{
Michael Ferrara $^{1} \quad$ Breeann Flesch ${ }^{2} \quad$ Ellen Gethner ${ }^{3}$ \\ Submitted: Nov 10, 2010; Accepted: Jul 29, 2011; Published: Aug 5, 2011 \\ Mathematics Subject Classification: 05C15, 05C25
}

\begin{abstract}
A coloring of the vertices of a graph $G$ is said to be distinguishing provided that no nontrivial automorphism of $G$ preserves all of the vertex colors. The distinguishing number of $G$, denoted $D(G)$, is the minimum number of colors in a distinguishing coloring of $G$. The distinguishing number, first introduced by Albertson and Collins in 1996, has been widely studied and a number of interesting results exist throughout the literature.

In this paper, the notion of distinguishing colorings is extended to that of listdistinguishing colorings. Given a family $L=\{L(v)\}_{v \in V(G)}$ of lists assigning available colors to the vertices of $G$, we say that $G$ is $L$-distinguishable if there is a distinguishing coloring $f$ of $G$ such that $f(v) \in L(v)$ for all $v$. The list-distinguishing number of $G, D_{\ell}(G)$, is the minimum integer $k$ such that $G$ is $L$-distinguishable for any assignment $L$ of lists with $|L(v)|=k$ for all $v$. Here, we determine the list-distinguishing number for several families of graphs and highlight a number of distinctions between the problems of distinguishing and list-distinguishing a graph.
\end{abstract}

Keywords: Distinguishing Coloring, List Coloring, List-Distinguishing Coloring

\section{Introduction}

A vertex coloring of a graph $G, f: V(G) \rightarrow\{1, \ldots, r\}$ is said to be $r$-distinguishing if no nontrivial automorphism of the graph preserves all of the vertex colors. The distinguishing

\footnotetext{
${ }^{1}$ Department of Mathematical and Statistical Sciences, University of Colorado Denver, Denver, CO 80217. michael.ferrara@ucdenver. edu.

${ }^{2}$ Mathematics Department, Western Oregon University, Monmouth, OR 97361. breeannmarie@gmail.com. Research partially supported by UCD GK12 project, NSF award \#0742434

${ }^{3}$ Department of Computer Science and Engineering, University of Colorado Denver, Denver, CO 80217. ellen.gethner@ucdenver.edu .
} 
number of a graph $G$, denoted $D(G)$, is the minimum integer $r$ such that $G$ has a $r$ distinguishing coloring and was first introduced by Albertson and Collins in [AC96].

In this paper we introduce the list-coloring analogue to the distinguishing problem. Given a family $L=\{L(v)\}_{v \in V(G)}$ of lists assigning available colors to the vertices of $G$, we say that $G$ is $L$-distinguishable if there is a distinguishing coloring $f$ of $G$ such that $f(v) \in L(v)$ for all $v$. The list-distinguishing number of $G$, written $D_{\ell}(G)$, is the smallest positive integer $k$ such that $G$ is $L$-distinguishable for any assignment $L$ of lists with $|L(v)|=k$ for all $v$. Since all of the lists can be identical, we observe that $D(G) \leq D_{\ell}(G)$.

In some cases, the list-distinguishing number can easily be shown to equal the distinguishing number. For example, it is not difficult to see that $D\left(K_{n}\right)=n=D_{\ell}\left(K_{n}\right)$ and $D\left(K_{n, n}\right)=n+1=D_{\ell}\left(K_{n, n}\right)$. In other cases, determining $D_{\ell}(G)$ is not nearly as simple, as the techniques needed to $k$-list-distinguish a graph can be significantly different from those used to distinguish a graph. This is especially apparent when considering those graphs $G$ with distinguishing number exactly 2. Such a graph $G$ necessarily has at least one nontrivial automorphism, and hence must have distinguishing number at least two. All that remains then is to demonstrate a 2-distinguishing coloring of $G$, which, to be clear, is often a highly nontrivial task. However, when one attempts to 2-list-distinguish the same $G$, it is not sufficient to simply demonstrate a coloring, as the lists assigned to $V(G)$ may be highly disparate in nature.

As an example, consider the problem of distinguishing $C_{n}$ for $n \geq 6$, which was shown in [AC96] to be 2. The automorphism group of $C_{n}$ is nontrivial, so clearly $D\left(C_{n}\right) \geq 2$, and the coloring in Figure 1, which generalizes to a coloring of $C_{n}$, suffices to complete the proof.

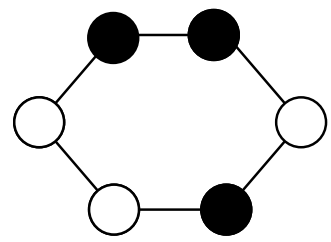

Figure 1: A distinguishing coloring of $C_{6}$.

The next proposition gives some indication of the increased difficulty one might encounter when considering list-distinguishing colorings.

Proposition 1. For $n \geq 6, D_{\ell}\left(C_{n}\right)=D\left(C_{n}\right)=2$.

Proof. Assign a list $L(v)$ of two colors to each $v \in V\left(C_{n}\right)$. If $|\bigcup L(v)|=2$, then the lists are identical, so we can color the vertices in a manner identical to the traditional distinguishing coloring, and we are done. Assume then that $|\bigcup L(v)| \neq 2$ and let $c$ be the color that appears in the fewest lists. Choose a vertex with $c$ in its list, label it $v_{1}$, and then continue labeling the vertices consecutively in numerical order. Color $v_{1}$ with $c$. Moving forward, no other vertex will receive color $c$, so that $v_{1}$ will be fixed by every color-preserving automorphism. Since $c$ appears in the fewest lists, there are at least $\frac{n}{3}$ 
vertices that do not have a $c$ in their list. Choose a vertex, $v_{i}$, such that $c \notin L\left(v_{i}\right)$ and is not antipodal to $v_{1}$. Consider the vertex $v_{n+2-i}$, which is the image of $v_{i}$ under the unique non-identity automorphism that fixes $v_{1}$. There exists $x \in L\left(v_{n+2-i}\right)$ such that $x \neq c$; color $v_{n+2-i}$ with $x$. Now there is a $y \in L\left(v_{i}\right)$ such that $y \neq x$ and we know that $y \neq c$, so color $v_{i}$ with $y$. Color the remaining vertices with any element from their list that is not $c$, as in Figure 2. Since any non-identity color-preserving automorphism must map $v_{1}$ to $v_{1}, v_{i}$ must map to $v_{n+2-i}$, but they are colored differently. Therefore the coloring is list-distinguishing, and $D_{\ell}\left(C_{n}\right)=D\left(C_{n}\right)=2$.

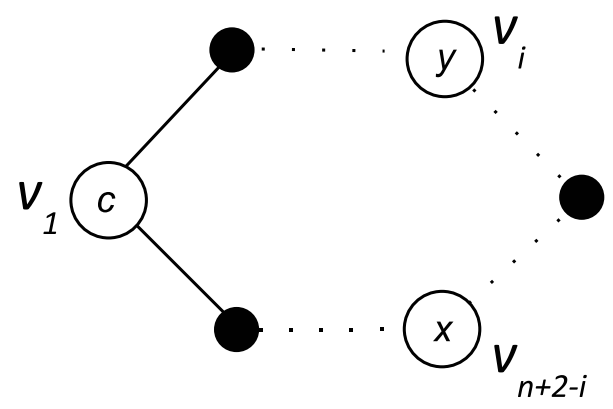

Figure 2: A 2-list-distinguishing coloring of $C_{n}$. The color $c$ appears only on $v_{1}$, and the colors $x$ and $y$ are distinct.

While Proposition 1 indicates how different the problems of determining $D(G)$ and $D_{\ell}(G)$ may be, at times it is possible to utilize existing techniques to obtain results on $D_{\ell}$. For instance, Brooks-type theorems for the (traditional) distinguishing number were given independently by Klavžar, Wong, and Zhu [KWZ06] and Collins and Trenk [CT06]. We are able to modify the approach from [KWZ06] to give a Brooks-type theorem for list-distinguishing colorings. Interestingly, we are able to show that the traditional distinguishing problem is precisely what prevents the exceptional graphs $K_{n}, K_{n, n}$ and $C_{5}$ from being $\Delta$-list-distinguishable.

Proposition 2. Let $G$ be a connected graph and let $\mathcal{L}=\{L(v)\}$ be an assignment of lists of size $\Delta(G)$ to $V(G)$. Then $G$ can be $\mathcal{L}$-distinguished unless $G$ is one of $K_{n}, K_{n, n}$, or $C_{5}$ and $|\bigcup L(v)|=\Delta(G)$. In these exceptional cases, $G$ can be colored from any assignment of lists of length $\Delta(G)+1$.

Our proof relies on the following lemma from [KWZ06].

Lemma 1. Suppose $(G, \ell)$ is a connected, vertex-colored graph such that $\ell(v)$ is the color for $v \in V(G)$. Let every vertex of the set $X \subseteq V(G)$ be fixed by every automorphism of $(G, \ell)$. Let $x \in X$ and set $S=N_{G}(x) \backslash X$. If $\ell(u) \neq \ell(v)$ for any pair of distinct vertices $u$ and $v$ in $S$, then every vertex of $S$ is fixed by every automorphism of $(G, \ell)$. 
Proof. (of Proposition 2) Assign list $L(v)$ to each vertex $v \in V(G)$ such that $|L(v)|=$ $\Delta(G)$, and assume $|\bigcup L(v)| \neq \Delta(G)$. Since $G$ is connected, there exist two vertices, $x$ and $y$, such that $L(x) \neq L(y)$ and $x y \in E(G)$. Let $c_{x} \in L(x)-L(y)$, and color $x$ with $c_{x}$. Going forward, no other vertex but $x$ will receive color $c_{x}$, assuring that $x$ will be fixed by every color-preserving automorphism of $G$. Construct a breadth first search spanning tree of $G$ rooted at $x$. Since $\left|N_{G}(x)\right| \leq \Delta(G)$, we can color each vertex $w \in N_{G}(x)-y$ with a unique color, $c_{w} \in L(w)$, such that $c_{w} \neq c_{x}$. Since $|L(y)|=\Delta(G)$ and $c_{x} \notin L(y)$, there exists a color, $c_{y} \in L(y)$ such that $c_{y} \neq c_{w}$ for all $w \in N_{G}(x)-y$; color $y$ with $c_{y}$. From here each vertex has at most $\Delta(G)-1$ children in the spanning tree. Therefore we can color each sibling of the spanning tree uniquely from its list, never using the color $c_{x}$. By an inductive application of Lemma 1, the coloring so constructed is a list-distinguishing coloring of $G$.

It remains to consider when $|\bigcup L(v)|=\Delta(G)$, which is the same as distinguishing coloring. Therefore from [CT06] and [KWZ06], we have that if $G$ is $K_{n}, K_{n, n}$, or $C_{5}$ then $D_{\ell}(G)=\Delta(G)+1$ and if not then $D_{\ell}(G)=\Delta(G)$.

Proposition 2 immediately yields the following Brooks-type result.

Theorem 1. Let $G$ is a graph with maximum degree $\Delta$. Then $D_{\ell}(G) \leq \Delta$ unless $G$ is one of $C_{5}, K_{n}$ or $K_{n, n}$, in which case $D_{\ell}(G)=\Delta+1$.

\section{Dihedral Groups}

Let $G$ be a graph and let $\Gamma$ be a group. If $A u t(G)$, the automorphism group of $G$, is isomorphic to $\Gamma$ then we will say that $G$ realizes $\Gamma$. Given a group element $g \in A u t(G)$ and a vertex $v$ in $G$, we let $v g$ denote the result of the action of $g$ on $v$. For a vertex $v$ in $G$, we let $S t(v)=\{h \in \Gamma \mid v h=v\}$ and $O(v)=\{b \in V(G) \mid v=b h$ for some $h \in \Gamma\}$ be the stabilizer and orbit of $v$ under the action of $\Gamma$ on $G$. The order of the orbit of $v$ under the action of $\Gamma$ on $G$ is $|A u t(G)| /|S t(v)|$. Also, we let $N_{G}(x)$ denote the set of vertices adjacent to $x$.

We let $D_{n}$ denote the dihedral group of order $2 n$, which is the group of symmetries of a regular $n$-gon. Throughout this section, we use the standard presentation $D_{n}=<$ $\sigma_{n}, \tau_{n} \mid \sigma_{n}^{2 n}=\tau_{n}^{2}=e, \sigma_{n} \tau_{n}=\tau_{n} \sigma_{n}^{-1}>$ where $\sigma_{n}$ and $\tau_{n}$ denote the appropriate rotation and reflection of the $n$-gon, respectively and $e$ denotes the identity element. We will frequently write $\tau_{n}=\tau$ and $\sigma_{n}=\sigma$ if the context is clear. When there is no danger of ambiguity, we will also let $e$ denote the identity element of an arbitrary group $\Gamma$.

In this section, we study the list-distinguishing number of graphs realizing $D_{n}$ for some $n \geq 3$. It is clear that $C_{n}$ realizes $D_{n}$, but there are many other graphs that realize the dihedral group (see Figure 3).

Albertson and Collins [AC96] proved that if $G$ realizes $D_{n}$ then $D(G)=2$ unless $n=\{3,4,5,6,10\}$ in which case $D(G)$ is either 2 or 3 . The main result of this section is as follows. 

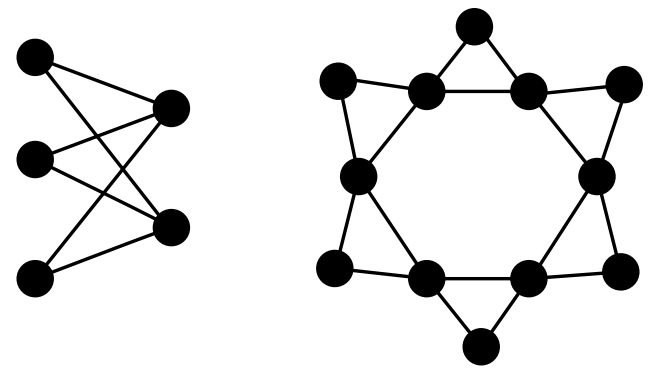

Figure 3: Two graphs that realize $D_{6}$.

Theorem 2. Let $G$ be a graph realizing $D_{n}$. Then $D(G)=D_{\ell}(G)$.

One interesting, and useful, consequence of our proof of Theorem 2 is that we determine precisely those graphs that realize $D_{n}$ and have (traditional) distinguishing number 3 .

We also point out at this time that the lemmata developed here to prove Theorem 2 appear nearly identical to those utilized to prove the corresponding theorem in [AC96]. However, the techniques used here frequently vary greatly from those in [AC96], illustrating further the distinctions between the distinguishing and list-distinguishing numbers.

Lemma 2. Let $G$ realize group $\Gamma$ and suppose $u_{1}, \ldots, u_{t}$ are vertices from different vertex orbits of $G$. If $\bigcap_{i=1}^{t} S t\left(u_{i}\right)=\{e\}$, then $D_{\ell}(G)=2$.

Proof. For each $u_{i}$, select a color $c_{i} \in L\left(u_{i}\right)$ and then color each other vertex in $O\left(u_{i}\right)$ with any color other than $c_{i}$. Let $g$ be any nonidentity element in $\Gamma$. Since $\bigcap_{i=1}^{t} \operatorname{St}\left(u_{i}\right)=\{e\}$, at least one $u_{i}$ is not fixed by $G$. Since $u_{i} g$ is not colored $c_{i}$, this is a 2-list-distinguishing coloring.

The following lemmas, the first of which appears in [AC96], will be useful as we proceed.

Lemma 3. Let $G$ realize $D_{n}$, and suppose that $G$ has $t$ vertex orbits. If $u_{1}, \ldots, u_{t}$ are vertices from the $t$ different vertex orbits of $G$, then

$$
\langle\sigma\rangle \cap S t\left(u_{1}\right) \cap \cdots \cap S t\left(u_{t}\right)=\{e\} .
$$

Lemma 4. Let $G$ realize $D_{n}$. If there is a vertex $u$ in $G$ such that $S t(u)=\left\langle\sigma^{j}\right\rangle$ then $D_{\ell}(G)=2$.

Proof. The proof of this lemma is identical to the proof of the corresponding lemma in [AC96]. Let $u_{1}, \ldots, u_{t}$ be vertices from each of the different orbits of $G$. Then $\bigcap_{i} S t\left(u_{i}\right) \subseteq$ $S t(u)$, which implies that $\bigcap_{i} S t\left(u_{i}\right)=\{e\}$, completing the proof by Lemma 2 .

At this point we begin to more seriously modify the techniques from [AC96] in order to better fit our list-coloring framework.

Lemma 5. Let $G$ realize $D_{n}$ and let $u$ be a vertex in $G$ such that $S t(u)$ is either $\left\langle\sigma^{j}, \tau \sigma^{i}\right\rangle$ or $\left\langle\tau \sigma^{i}\right\rangle$. If $|O(u)| \geq 6$, then $O(u)$ is 2-list-distinguishable. 
Proof. Regardless of whether $S t(u)=\left\langle\sigma^{j}, \tau \sigma^{i}\right\rangle$ or $S t(u)=\left\langle\tau \sigma^{i}\right\rangle$, we have that $O(u)=$ $\left\{u, u \sigma, u \sigma^{2}, \ldots, u \sigma^{j-1}\right\}$ for some $j$, and hence we assume that $j \geq 6$. Consider the set $A=\left\{u, u \sigma^{2}, u \sigma^{3}\right\}$ and select a color $c_{u} \in L(u)$. If possible, color $u \sigma^{2}$ and $u \sigma^{3}$ with $c_{u}$ as well. We will demonstrate that it is possible to extend this to a 2-distinguishing coloring without using $c_{u}$ on any vertex in $V(G)-A$.

Case 1: All three vertices in $A$ are colored with $c_{u}$.

We proceed by coloring the vertices in $V(G)-A$ using any color in their respective lists except $c_{u}$. Any automorphism $g$ in $D_{n}$ that fixes this coloring of $O(u)$ must permute the vertices in $A$, and specifically must map $u$ to some element of $A$. Therefore $g$ must lie in $S t(u), S t(u) \sigma^{2}$ or $S t(u) \sigma^{3}$. As was demonstrated in [AC96], the only automorphisms from these sets that permute $A$ actually fix all of $O(u)$. Thus this is a 2-list-distinguishing coloring of $O(u)$.

Case 2: The vertex $u$ is the only one in $A$ colored with $c_{u}$.

Suppose that $S t(u)=\left\langle\sigma^{j}, \tau \sigma^{i}\right\rangle$. Then $j$ divides $n$, so the assumption that $j \geq 6$ implies that $u \sigma^{2} \neq u \sigma^{n-2}$, which also holds when $S t(u)=\left\langle\tau \sigma^{i}\right\rangle$. In either case, we extend the coloring of $A$ by first assigning the vertex $u \sigma^{n-2}$ any color $c \neq c_{u}$ in $L\left(u \sigma^{n-2}\right)$. Since we utilize the color $c_{u}$ on the vertices in $A$ wherever possible, and $u$ is the only vertex of $G$ that receives color $c_{u}$, we conclude that $c_{u} \notin L\left(u \sigma^{2}\right)$. Therefore there is a color $c^{\prime}$ in $L\left(u \sigma^{2}\right)$, different from both $c$ and $c_{u}$. Color $u \sigma^{2}$ using color $c^{\prime}$ and color the remaining vertices of $G$ with any color aside from $c_{u}$ in their respective lists.

We now show that this coloring distinguishes $O(u)$. Since $u$ is the unique vertex of color $c_{u}$, any color-preserving automorphism $g \in D_{n}$ must lie in $S t(u)$. Then either $g=\sigma^{d j}$ or $g=\tau \sigma^{i+d j}$, with $d$ being necessarily zero when $S t(u)=\left\langle\tau \sigma^{i}\right\rangle$. Note that $\sigma^{d j}$ fixes $O(u)$ and that, for any $d, \tau \sigma^{i+d j}$ takes $u \sigma^{2}$ to $u \sigma^{n-2}$. Since we have constructed our coloring so that $u \sigma^{2}$ and $u \sigma^{n-2}$ have different colors, this is a 2-list-distinguishing coloring of $O(u)$.

Case 3: The vertices $u$ and $u \sigma^{2}$ are the only ones in $A$ colored with $c_{u}$.

As above, if $S t(u)=\left\langle\sigma^{j}, \tau \sigma^{i}\right\rangle$, then $j$ divides $n$. Consequently, the assumption that $j \geq 6$ implies that $u \sigma^{3} \neq u \sigma^{n-1}$, which again also holds when $S t(u)=\left\langle\tau \sigma^{i}\right\rangle$. In this case, we extend the coloring of $u$ and $u \sigma^{2}$ in a similar manner to Case 1, with two exceptions. We make no special color assignment to $u \sigma^{n-2}$, save the standard assumption that it does not receive color $c_{u}$. Instead, we assign different colors to the vertices $u \sigma^{3}$ and $u \sigma^{n-1}$ such that neither vertex is colored with $c_{u}$. As above, this is possible since $c_{u} \notin L\left(u \sigma^{3}\right)$, or else we would have used it to color $u \sigma^{3}$.

Now, every automorphism $g \in D_{n}$ that preserves the colors on $O(u)$ must either fix or interchange $u$ and $u \sigma^{2}$. As discussed in the previous case, any element of $S t(u)$ will either fix all of $O(u)$ or will map $u \sigma^{2}$ to $u \sigma^{n-2}$. Thus as $u \sigma^{n-2}$ is not colored with $c_{u}$, any color preserving automorphism $g$ must interchange $u$ and $u \sigma^{2}$. This implies that $g$ has the form $g^{\prime} \sigma^{2}$, where $g^{\prime}$ is an element of $S t(u)$. Specifically, either $g^{\prime}=\sigma^{d j}$ or $g=\tau \sigma^{i+d j}$ for some integer $d$, with $d=0$ if $S t(u)=\left\langle\tau \sigma^{i}\right\rangle$. In either case, $g^{\prime} \sigma^{2}$ takes $u \sigma^{3}$ to $u \sigma^{n-1}$, implying that this coloring 2-list-distinguishes $O(u)$.

Case 4: The vertices $u$ and $u \sigma^{3}$ are the only ones in $A$ colored with $c_{u}$. 
Suppose first that $S t(u)=\left\langle\tau \sigma^{i}\right\rangle$. We wish, as above, to extend our coloring of $A$ to a list-distinguishing coloring of $O(u)$ in which no vertices aside from $u$ and $u \sigma^{3}$ receive color $c_{u}$. Any element $g \in D_{n}$ that fixes such a coloring must either stabilize $u$ or exchange $u$ and $u \sigma^{3}$. Consequently, either $g=\tau \sigma^{i}$ or $g=\tau \sigma^{i+3}$, so consider the outcomes when these elements are applied to $A$ :

$$
\begin{aligned}
& A \tau \sigma^{i}=\left\{u, u \sigma^{n-2}, u \sigma^{n-3}\right\} \\
& A \tau \sigma^{i+3}=\left\{u \sigma^{3}, u \sigma, u\right\}
\end{aligned}
$$

Therefore, we would like to choose colors $x \in L(u \sigma)$ and $y \in L\left(u \sigma^{n-2}\right), x, y \neq c_{u}$ and choose a color $z \neq x, y$ from $L\left(u \sigma^{2}\right)$. We would then extend our coloring of $A$ by assigning these colors to their respective vertices and then coloring the remaining vertices in $G$ using any color except $c_{u}$. This is possible unless all of the following hold: $L(u \sigma)=$ $\left\{c_{u}, x\right\}, L\left(u \sigma^{n-2}\right)=\left\{c_{u}, y\right\}$ and $L\left(u \sigma^{2}\right)=\{x, y\}$. In this case, suppose $L(u)=\left\{c_{u}, c_{u}^{\prime}\right\}$. We will then recolor $u$ with $c_{u}^{\prime}$ and again color $u \sigma^{2}$ and $u \sigma^{3}$ with these colors if possible. Then, the analysis conducted thus far assures that we can construct a 2-list-distinguishing coloring of $O(u)$, as we cannot have $L(u \sigma)=\left\{c_{u}^{\prime}, x\right\}, L\left(u \sigma^{n-2}\right)=\left\{c_{u}^{\prime}, y\right\}$ and $L\left(u \sigma^{2}\right)=$ $\{x, y\}$ for $x=y$, and this was the only obstacle preventing us from constructing the desired coloring when $S t(u)=\left\langle\tau \sigma^{i}\right\rangle$.

Hence we assume that $S t(u)=\left\langle\sigma^{j}, \tau \sigma^{i}\right\rangle$. In this case, $j$ divides $n$, and we may assume that $n>j \geq 6$. Again, we wish to extend our coloring of $A$ to a distinguishing coloring of $G$ in which no vertices aside from $u$ and $u \sigma^{3}$ receive color $c_{u}$. If $g \in D_{n}$ fixes such a coloring then either $g \in S t(u)$ or $g=g^{\prime} \sigma^{3}$ for some $g^{\prime} \in S t(u)$. Thus for some $d \geq 0$, $g=\sigma^{d j}$, either $\sigma^{d j+3}, \tau \sigma^{i+d j}$ or $\tau \sigma^{i+d j+3}$. Applying each of these elements to $A$, we obtain

$$
\begin{aligned}
& A \sigma^{d j}=A \\
& A \sigma^{d j+3}=\left\{u \sigma^{3}, u \sigma^{5}, u \sigma^{6}\right\} \\
& A \tau \sigma^{d j+i}=\left\{u, u \sigma^{n-2}, u \sigma^{n-3}\right\} \\
& A \tau \sigma^{d j+i+3}=\left\{u \sigma^{3}, u \sigma, u\right\} .
\end{aligned}
$$

Since $j$ divides $n$ and $n$ is at least seven, $u \sigma^{n-3} \neq u \sigma^{3}$. Therefore $g=\tau \sigma^{d j+i}$ cannot preserve our proposed coloring. Additionally, $\sigma^{d j}$ fixes all of $O(u)$, so we need only consider the cases where $g=\sigma^{d j+3}$ or $g=\tau \sigma^{d j+i+3}$.

If $j>6$, then since $\sigma^{d j+3}$ takes $u \sigma^{3}$ to $u \sigma^{6}$ this choice of $G$ cannot distinguish $u$. Thus when $j>6$ is suffices to assume that $g=\tau \sigma^{i+d j}$ and thus we need only to distinguish $u \sigma$ from $u \sigma^{2}$ without assigning $c_{u}$ to $u \sigma$, which is clearly possible as we have assumed that $c_{u} \notin L\left(u \sigma^{2}\right)$.

Thus we assume that $j=6$ and that either $g=\sigma^{d j}$ or $g=\tau \sigma^{i+d j+3}$. We would like to choose colors $x \in L(u \sigma)$ and $y \in L\left(u \sigma^{5}\right), x, y \neq c_{u}$ and choose a color $z \neq x, y$ from $L\left(u \sigma^{2}\right)$. We would then extend our coloring of $A$ by assigning these colors to their respective vertices and then coloring the remaining vertices in $G$ using any color except $c_{u}$. As above, this is possible unless $L(u \sigma)=\left\{c_{u}, x\right\}, L\left(u \sigma^{5}\right)=\left\{c_{u}, y\right\}$ and $L\left(u \sigma^{2}\right)=\{x, y\}$. However, in this case we proceed by changing our initial coloring of $A$ by using the color 
$c_{u}^{\prime} \neq c_{u}$ in $L(u)$. As when $S t(u)=\left\langle\tau \sigma^{i}\right\rangle$, this allows us to construct a coloring that will distinguish $O(u)$, and therefore completes the proof.

The following lemma is a slight modification of the corresponding lemma in [AC96].

Lemma 6. Let $G$ realize $D_{n}$ and let $u$ be a vertex such that $u \in V(G)$ and $S t(u)=<$ $\sigma^{j}, \tau \sigma^{i}>$ or $<\tau \sigma^{i}>$. If $|O(u)| \geq 6$, then $D_{\ell}(G)=2$.

Proof. Assign lists of length two to each vertex in $G$. If $S t(u)=<\tau \sigma^{i}>$, then the intersection of the subgroups conjugate to $S t(u)$ is the identity. Applying Lemma 5, $O(u)$ is 2-list-distinguishable and thus by Lemma $2, D_{\ell}(G)=2$.

Therefore, assume $S t(u)=<\sigma^{j}, \tau \sigma^{i}>$. Since $O(u)$ is 2-list-distinguishable, we need only consider the automorphisms that act trivially on $O(u)$. These are the intersection of the stabilizers of vertices of $O(u)$, which is $\Lambda=<\sigma^{j}>$. The group action of $\Lambda$ on $G$ creates vertex orbits $U_{1}, U_{2}, . ., U_{t}$. From each orbit $U_{i}$ such that $\left|U_{i}\right|>1$, select a vertex $u_{i}$ and color it with any color $c_{i} \in L\left(u_{i}\right)$. Then color the remaining vertices of $U_{i}$ with any color other than $c_{i}$, construct a distinguishing coloring of $O(u)$ from its assigned lists and color all other uncolored vertices in $G$ with any color from their respective lists. If a nontrivial automorphism in $\Lambda$ fixes $u_{i}$, then it must fix all of $U_{i}$. Thus each $g \neq e$ in $\Lambda$ must move some $u_{i}$ to another vertex in its orbit, implying that the only color preserving automorphism is the identity. Consequently, this is a 2-list-distinguishing coloring of $G$, so $D_{\ell}(G)=2$.

Lemmas $2-5$ provide the necessary machinery to complete the proof of Theorem 2 , and we do so now.

Proof. (of Theorem 2) When $n>10$ there must be an orbit of order at least 6 , so it remains to show that the theorem holds when $n=3,4,5,6$ or 10 . Suppose first that $G$ realizes $D_{n}$, where $n=3$ or 5 and select a vertex $u$ with nontrivial orbit in $G$. By the above lemmas, we may assume that every vertex $u$ in $G$ has $S t(u)=\left\langle\tau \sigma^{i}\right\rangle$ or $\left\langle\sigma^{j}, \tau \sigma^{i}\right\rangle$. However, as $n$ is prime, if $\sigma^{j}$ fixes $u$, then $j=0$ or $j=1$. Consequently, we may assume that $j=0$, as $\left\langle\sigma, \tau \sigma^{i}\right\rangle=D_{n}$, and by assumption $u$ has a nontrivial orbit.

Note as well that if $\tau \sigma^{i}$ is in $S t(u)$, then $\tau$ is in $S t\left(u \sigma^{x}\right)$ where $2 x \equiv n-i(\bmod n)$. Such an $x$ exists for all $i$ when $n=3$ or 5 , so we may assume that $S t(u)=\langle\tau\rangle$. Then, since the orbit of $u$ is by assumption nontrivial, $O(u)=\left\{u, u \sigma, \ldots, u \sigma^{n-1}\right\}$ and the only element of $D_{n}$ that fixes all of $O(u)$ is $e$.

If $G$ has exactly one nontrivial orbit, then necessarily $D(G)=3$, and since this orbit is precisely $O(u)=\left\{u, u \sigma, \ldots, u \sigma^{n-1}\right\}$, it is straightforward to show that $D_{\ell}(G)=3$ as well. Suppose then that $G$ has vertices $u$ and $v$ with distinct nontrivial orbits and that we have also assigned lists of length 2 to the vertices of $G$. We may furthermore assume, via the above discussion, that $S t(u)=S t(v)=\langle\tau\rangle$. If it is not possible to color each vertex in $O(u)$ with a unique color, then each vertex in $O(v)$ must have list $L\left(u \sigma^{i}\right)=\left\{c_{1}, c_{2}\right\}$. We then color $u$ with color $c_{1}$ and color both $u \sigma$ and $u \sigma^{2}$ with color $c_{2}$. 
If $g$, a nonidentity element of $D_{n}$, fixes this coloring of $O(u)$, then $g \in \operatorname{St}(u)$. However, since $O(v)$ is nontrivial, $g$ must exchange two elements in $O(v)$, say $v \sigma^{i}$ and $v \sigma^{j}$. Assigning these vertices distinct colors from their respective lists serves to 2-list-distinguish $G$, and thus implies that $D(G)=D_{\ell}(G)=2$.

Next, let $G$ be a graph that realizes $D_{4}$ and furthermore assume that there is no vertex $u$ in $G$ such that $S t(u)=\left\langle\sigma^{j}\right\rangle$ or $|O(u)| \geq 6$. Lemmas 2 and 3 imply that if there are vertices $u$ and $v$ in distinct orbits of $G$ such that $\tau \sigma^{i} \in S t(u)$ but $\tau \sigma^{i} \notin S t(v)$, then $D(G)=D_{\ell}(G)=2$.

Suppose that $\tau$ stabilizes some element in every orbit of $G$ (the case where some other $\tau \sigma^{i}$ stabilizes an element in every orbit is handled similarly). Under our assumptions, every nontrivial orbit in $G$ must have order either two or four. If every nontrivial orbit has two elements, then $\tau$ stabilizes every vertex in $G$, a contradiction. Therefore, there is some vertex $u$ such that $S t(u)=\langle\tau\rangle$ and $O(u)=\left\{u, u \sigma, u \sigma^{2}, u \sigma^{3}\right\}$. If possible, color the orbit of $u$ in a manner consistent with the traditional distinguishing coloring of $C_{4}$, specifically, for some $i$, color $u \sigma^{i}$ and $u \sigma^{i+1}$ with the same color $c$ and then color the other two vertices in $O(u)$ with distinct colors other than $c$. Since no element of $D_{4}$ fixes all of $O(u)$, this would suffice to 2-list-distinguish $G$ and also shows that $D_{\ell}(G) \leq 3$. Thus we may assume that $L\left(u \sigma^{i}\right)=\left\{c_{1}, c_{2}\right\}$ for all $i$ implying that if $O(u)$ is the only nontrivial orbit of $G, D(G)=D_{\ell}(G)=3$.

Next, assume that there is some vertex $v$, not in $O(u)$, such that $O(v)$ is nontrivial and assign lists of length two to each vertex in $v$. We claim that it is possible to construct a distinguishing coloring of $v$ from these lists. If $|O(v)|=4$, then $O(v)=\left\{v, v \sigma, v \sigma^{2}, v \sigma^{3}\right\}$ and we may assume that $L\left(v \sigma^{i}\right)=\left\{c_{1}^{\prime}, c_{2}^{\prime}\right\}$ for all $i$. Without loss of generality, suppose that $\tau$ stabilizes $v$ (and therefore $v \sigma^{2}$ ). Color $u$ and $u \sigma$ with color $c_{1}, u \sigma^{2}$ and $u \sigma^{3}$ with color $c_{2}, v$ and $v \sigma^{2}$ with color $c_{1}^{\prime}$ and, finally, $v \sigma$ and $v \sigma^{3}$ with color $c_{2}^{\prime}$. Then the only automorphism that fixes the colors in both $O(u)$ and $O(v)$ is $e$, so this coloring 2-listdistinguishes $G$.

We may therefore suppose that $O(v)$, and every nontrivial orbit of $G$ aside from $O(u)$ has exactly two elements, and hence that $S t(v)=\left\langle\sigma^{2}, \tau\right\rangle$ (as, again, the case where $S t(v)=\left\langle\sigma^{2}, \tau \sigma^{j}\right\rangle$ is similar) and $O(v)=\{v, v \sigma\}$. We will color $u$ and $u \sigma$ with color $c_{1}$, $u \sigma^{2}$ and $u \sigma^{3}$ with color $c_{2}$ and color $v$ and $v \sigma$ with distinct colors. The only nonidentity automorphism of $G$ that preserves this coloring of $O(u)$ is $\tau \sigma^{3}$. However, this interchanges the elements of $O(v)$, which received different colors, so this coloring 2-list-distinguishes $G$.

Next we consider the penultimate case that $G$ realizes $D_{6}$. By the above lemmas, we may assume that there is no vertex $u$ in $G$ has $S t(u)=<\sigma^{j}>$ or $|O(u)| \geq 6$. If there exists a $u \in V(G)$ such that $S t(u)=<\tau \sigma^{i}>$, then $|O(u)|=6$, therefore we assume that every vertex $u$ in $G$ has $S t(u)=<\sigma^{j}, \tau \sigma^{i}>$. Given this stabilizer, it is not hard to show that for every vertex $u$ in $G,|O(u)|=1,2$ or 3 . This implies that $G$ can easily be 3 -listdistinguished, as it is possible to all of the vertices in a given orbit with distinct colors. Furthermore, if there is no orbit of order 3, then it is not difficult to 2-list-distinguish $G$.

Therefore, let us first assume there is exactly one orbit of order 3. Specifically, let $u$ be 
a vertex such that $|O(u)|=3$ and observe that necessarily $O(u)=\left\{u, u \sigma, u \sigma^{2}\right\}$. Suppose first that for each $x \in O(u)$ there is some $\phi_{x} \in \operatorname{Aut}(G) \cap S t(x)$ such that the following hold:

1. $\phi_{x}$ interchanges the two vertices in $O(u)-x$, and

2. $\phi_{x}$ fixes all of $V(G)-O(u)$.

In this case, we cannot 2-distinguish $G$ with any 2-coloring, as without loss of generality both $u \sigma$ and $u \sigma^{2}$ will receive the same color. However, then the automorphism $\phi_{u}$ described above is nontrivial and color-preserving, regardless of how the remainder of $G$ is colored.

If there is some $x \in O(u)$ for which no such $\phi_{x}$ exists, then we claim that $G$ is 2-listdistinguishable (and hence 2-distinguishable). In this case, we assign distinct colors to all pairs of vertices lying in orbits of order two and also color all vertices of $O(u)$ with distinct colors, if this is possible. If it is not possible to color $O(u)$ in this way, then each vertex in $O(u)$ must be assigned identical lists, say $\left\{c_{1}, c_{2}\right\}$. We then color $x$ with $c_{1}$ and the vertices in $O(u)-x$ with $c_{2}$. Due to the assumptions that $O(u)$ is the unique orbit of order three and that there is no $\phi_{x}$ as described above, any nontrivial automorphism $g \in \operatorname{Aut}(G)$ that preserves this coloring of $O(u)$ must interchange the elements of an orbit of order two. However, all such orbits have been colored with distinct colors, so $g$ is not color-preserving. It follows that $D_{\ell}(G)=2$

Suppose then that $G$ has more than one orbit of order 3, and assign lists of order 2 to the vertices of $G$. Choose vertices $u$ and $v$ in distinct orbits such that $|O(u)|=|O(v)|=3$. If it is not possible to color each vertex in $O(u)$ with a distinct color, then each vertex in $O(u)$ must have the same list, $\left\{c_{1}, c_{2}\right\}$. We then color $u$ with $c_{1}$ and the other two vertices with color $c_{2}$. If $g$, a nonidentity element of $D_{6}$, fixes this coloring of $O(u)$, then $g \in S t(u)$. However, since $|O(v)|=3, g$ must exchange two elements in $O(v)$. Assigning these vertices distinct colors from their respective lists serves to 2 -list-distinguish $G$. Therefore, $D(G)=D_{\ell}(G)=2$.

Suppose lastly that $G$ realizes $D_{10}$ and that there is no vertex $u$ in $G$ such that $S t(u)=<$ $\sigma^{j}>$ or $|O(u)| \geq 6$. If there exists a $u \in V(G)$ such that $S t(u)=<\tau \sigma^{i}>$, then $|O(u)|=10$, therefore we may assume that every vertex $u$ in $G$ has $S t(u)=<\sigma^{j}, \tau \sigma^{i}>$. Given this stabilizer, it is not hard to show that for every vertex $u$ in $G,|O(u)|=1,2$ or 5 .

Furthermore, as every orbit of order 5 in $V(G)$ has the form $O(u)=\left\{u, u \sigma, \ldots, u \sigma^{4}\right\}$ and stabilizer $\left\langle\sigma^{5}, \tau \sigma^{j}\right\rangle$ for some $j$, it is not difficult to see that the action of $D_{10}$ on any such orbit of order five can be viewed precisely as the action of $D_{5}$ on the vertices of $C_{5}$. We observe that this implies that $G$ is 3-list-distinguishable, as then we may color each orbit of order five such that three vertices receive distinct colors and also color the orbits of order two so that both vertices receive distinct colors. Keeping in mind the action of $\operatorname{Aut}(G)$ on $O(u)$, we can see that this is a distinguishing coloring. 
Again we can easily 2-list-distinguish $G$ if there are no orbits of order 5, so we first assume there is exactly one orbit of order 5. Specifically, let $u$ be a vertex such that $|O(u)|=5$ and recall that necessarily $O(u)=\left\{u, u \sigma, \ldots, u \sigma^{4}\right\}$. Suppose as well for every $x \in O(u)$ there is some $\phi_{x} \in S t(x)$ such that the following hold:

1. $\phi_{x}$ interchanges the pairs $\left(x \sigma, x \sigma^{4}\right)$ and $\left(x \sigma^{2}, x \sigma^{3}\right)$, and

2. $\phi_{x}$ fixes all of $V(G)-O(u)$.

As in the previous case, we claim that the existence of these $\phi_{x}$ implies that $G$ is not 2-distinguishable. In any 2-coloring of $O(u)$, there is some vertex $x$ such that the pairs $\left(x \sigma, x \sigma^{4}\right)$ and $\left(x \sigma^{2}, x \sigma^{3}\right)$ are monochromatic. However, then the automorphism $\phi_{x}$ described above is nontrivial and color-preserving, regardless of how the remainder of $G$ is colored. It follows that $G$ is not 2-list-distinguishable and, by our above observation, that $G$ must therefore be 3-list-distinguishable.

Suppose therefore, without loss of generality, that there is no such $\phi_{u} \in \operatorname{Aut}(G)$ and assign lists of length two to $V(G)$. We color each orbit of length two with distinct colors, and if possible assign distinct colors to three vertices in $O(u)$. If it is not possible to color three vertices with distinct colors, then each vertex in $O(u)$ must have the same list, $\left\{c_{1}, c_{2}\right\}$. We will then color $u$ with color $c_{1}$ and color $u \sigma, u \sigma^{2}, u \sigma^{3}$ and $u \sigma^{4}$ with color $c_{2}$. Given the action of $D_{10}$ on $O(u)$, the only nontrivial automorphisms that would fix this coloring of $O(u)$ necessarily fixes $u$ and exchanges the pairs $\left(u \sigma, u \sigma^{4}\right)$ and $\left(u \sigma^{2}, u \sigma^{3}\right)$. By the assumption that there is no $\phi_{u}$ satisfying conditions (1) and (2), such an automorphism must exchange the vertices in an orbit of order two and is therefore not color-preserving. It follows that $G$ is 2-list distinguishable.

Now assume there is more than one orbit of order 5 and assign lists of length 2 to the vertices of $G$. Choose vertices $u$ and $v$ in distinct orbits such that $|O(u)|=|O(v)|=5$. If it is possible to color three vertices in $O(u)$ with distinct colors, then such a coloring can be extended to a 2-list-coloring of $G$. If it is not possible to color three vertices with distinct colors, then again each vertex in $O(u)$ must have the same list, $\left\{c_{1}, c_{2}\right\}$. We then color two vertices, $v$ and $w$, with $c_{1}$ and the rest with color $c_{2}$. If $g$, a nonidentity element of $D_{10}$, fixes this coloring of $O(u)$, then either $g \in S t(v) \cap S t(w)$ or $g$ interchanges $v$ and $w$. However, since $|O(v)|=5, g$ must exchange two elements in $O(v)$. Assigning these vertices distinct colors from their respective lists serves to 2 -list-distinguish $G$. Therefore, $D(G)=D_{\ell}(G)=2$.

As a consequence of the proof of Theorem 2, we obtain the following.

Theorem 3. Let $G$ be a graph realizing $D_{n}$ such that $V(G)$ has no orbit of order greater than five, and also such that $G$ does not satisfy the hypotheses of Lemma 2 or Lemma 4. Then $D(G)=3$ if and only if $G$ has exactly one nontrivial orbit $O$ satisfying one of the following:

$$
\text { 1. } n \in\{3,4,5\} \text { and }|O|=n \text {, or }
$$


2. $n=6,|O|=3$, and for each $x \in O$ there is some $\phi_{x} \in S t(x)$ such that the following hold:

(a) $\phi_{x}$ interchanges the two vertices in $O(u)-x$, and

(b) $\phi_{x}$ fixes all of $V(G)-O(u)$, or

3. $n=10,|O|=5$, and for each $x \in O$ there is some $\phi_{x} \in S t(x)$ such that the following hold:

(a) $\phi_{x}$ interchanges the pairs $\left(x \sigma, x \sigma^{4}\right)$ and $\left(x \sigma^{2}, x \sigma^{3}\right)$, and

(b) $\phi_{x}$ fixes all of $V(G)-O(u)$.

\section{Cartesian Products of Graphs}

We use the standard definition for Cartesian products of graphs $G$ and $H$, (for example see [Wes01]) which we denote by $G \square H$. A fiber $G_{i}^{\left(g_{1}, \ldots, g_{k}\right)}$ of $G_{1} \square \ldots \square G_{k}$ is the subgraph induced by the vertex set $\left\{\left(g_{1}, \ldots, x, \ldots, g_{k}\right) \mid x \in G_{i}\right\}$.

The automorphism group of Cartesian products is well understood. If $G=\left(G_{1} \square G_{2}\right)$ and $\phi \in \operatorname{Aut}\left(G_{1}\right)$, then $\phi^{\prime}: V(G) \rightarrow V(G)$ defined by $\phi^{\prime}:\left(u_{i}, v_{j}\right) \mapsto\left(\phi u_{i}, v_{j}\right)$ is an automorphism of $G$. If $G_{1} \cong G_{2}$, then $\alpha:\left(u_{i}, v_{j}\right) \mapsto\left(v_{j}, u_{i}\right)$ is also an automorphism of $G$. In fact, the automorphisms of a factor and the exchange of isomorphic factors generate Aut $(G)$ [Imr67].

We now turn our attention to the Cartesian product of two cycles, also known as the toroidal grid. We label a vertex of $C_{n} \square C_{m}$ as $\left(u_{i}, v_{j}\right)$ if it is in fibers $C_{n}^{i}$ and $C_{m}^{j}$, but for simplicity, we denote $L((u, v))$ as $L(u, v)$. We also define $S_{c}(G)=\{v \in V(G) \mid c \in L(v)\}$ and will write $S_{c}(G)=S_{c}$ if the context is clear. If $n \neq m$, the automorphism group of $C_{n} \square C_{m}$ is generated by the Cartesian product of the generators of $C_{n}$ and $C_{m}$. This leads us to the following elementary lemma, given without proof, about the action of an automorphism of a cycle factor $C_{n}$, which will be used in subsequent proofs.

Lemma 7. Let $G=\left(C_{n} \square C_{m}\right)$ such that $n \neq m$. Let $\phi \in \operatorname{Aut}\left(C_{n}\right), \psi \in \operatorname{Aut}\left(C_{m}\right)$ and $\phi^{\prime} \in \operatorname{Aut}\left(C_{n} \square C_{m}\right)$ such that $\phi^{\prime}:\left(u_{i}, v_{j}\right) \mapsto\left(\phi u_{i}, \psi v_{j}\right)$. If $\phi^{\prime}:\left(u_{i}, v_{j}\right) \mapsto\left(u_{k}, \psi v_{j}\right)$, then $\phi^{\prime}:\left(u_{i}, v_{p}\right) \mapsto\left(u_{k}, \psi v_{p}\right)$ for all $p \in\{1, \ldots, m\}$.

The distinguishing number of Cartesian products has been extensively studied. In [BC04] Bogstad and Cowen determined that $D\left(Q_{d}\right)=2$ if $d \geq 4$ for the $d$-dimensional hypercube, $Q_{d}$. Using the motion lemma, a probabilistic result of Russell and Sundaram [RS98], Albertson extended Bogstad and Cowen's result by showing that for an arbitrary graph $G, D\left(G^{r}\right)=2$ if $r \geq 4$ [Alb05]. This result was improved by Klavžar and Zhu in [KZ07] and was further strengthened by Imrich and Klavžar who demonstrated that $D\left(G^{r}\right)=2$ when $r \geq 2$ and $G \neq K_{2}, K_{3}$ [IK06]. Other interesting results about distinguishing Cartesian products of graphs appear in [Bou09] and [CHK10]. 
To prove that $D\left(C_{n} \square C_{m}\right)=D_{\ell}\left(C_{n} \square C_{m}\right)$ for all $n$ and $m$, we use the following lemma, which says that if we can 2-list-distinguish any fiber in a specific way then the entire graph can be 2-list-distinguished.

Lemma 8. Let $G=C_{n} \square C_{m}$, and assign a list $L\left(u_{i}, v_{j}\right)$ of size two to each $\left(u_{i}, v_{j}\right) \in$ $V\left(C_{n} \square C_{m}\right)$. If there exists a fiber that can be L-list-distinguished with a vertex that has a unique color in that fiber, then $G$ can be L-list-distinguished.

Proof. Let $G=C_{n} \square C_{m}$, and assign a list $L\left(u_{i}, v_{j}\right)$ such that $\left|L\left(u_{i}, v_{j}\right)\right|=2$ to each $\left(u_{i}, v_{j}\right) \in V\left(C_{n} \square C_{m}\right)$. Assume we can color $C_{n}^{1}$ so that color $c_{1}$ is used on the vertex $\left(u_{1}, v_{1}\right)$ but on no other vertex of $C_{n}^{1}$.

Case 1: Suppose $V\left(C_{n}^{2}\right) \subseteq S_{c_{1}}$.

Color each vertex $\left(u_{i}, v_{2}\right) \in C_{n}^{2}$ with $c_{1}$. Assign list $L^{\prime}\left(u_{x}, v_{y}\right)=L\left(u_{x}, v_{y}\right)-c_{1}$ to all uncolored vertices, and $L^{\prime}$-list-color these vertices arbitrarily.

Case 2: There exist vertices $\left(u_{i}, v_{2}\right), i \neq 1$ and $\left(u_{1}, v_{j}\right), j \neq 1$ such that $\left(u_{i}, v_{2}\right),\left(u_{1}, v_{j}\right)$ $\notin S_{c_{1}}$.

For all uncolored vertices, assign list $L^{\prime}\left(u_{x}, v_{y}\right)=L\left(u_{x}, v_{y}\right)-c_{1}$. Color $\left(u_{i}, v_{m}\right)$ with some $c_{2} \in L^{\prime}\left(u_{i}, v_{m}\right)$, color $\left(u_{i}, v_{2}\right)$ with some $c_{3} \in L^{\prime}\left(u_{i}, v_{2}\right)$ such that $c_{3} \neq c_{2}$, and $L^{\prime}$ list-color the rest of the vertices. Since they are the only two fibers with a vertex colored $c_{1}, C_{n}^{1}$ must map to $C_{m}^{1}$ in any non-trivial color-preserving automorphism; furthermore, $C_{n}^{1}$ has been list-distinguished, so there is at most one automorphism, $\alpha$, that could map $C_{n}^{1}$ to $C_{m}^{1}$. Assume that $\alpha\left(u_{i}, v_{1}\right) \mapsto\left(u_{1}, v_{j}\right)$, and let the vertex $\left(u_{i}, v_{1}\right)$ be colored $c_{k}$. The vertex $\left(u_{1}, v_{j}\right) \notin S_{c_{1}}$, so recolor $\left(u_{1}, v_{j}\right)$ with $c_{a} \in L^{\prime}\left(u_{1}, v_{j}\right)$ such that $c_{a} \neq c_{k}$.

Case 3: There exists a vertex $\left(u_{i}, v_{2}\right), i \neq 1$, such that $\left(u_{i}, v_{2}\right) \notin S_{c_{1}}$, and $\left(u_{1}, v_{j}\right) \in S_{c_{1}}$ for all $j \neq 1$.

Color $\left(u_{1}, v_{2}\right)$ with $c_{1}$, and assign list $L^{\prime}\left(u_{x}, v_{y}\right)=L\left(u_{x}, v_{y}\right)-c_{1}$ to all uncolored vertices. Let vertex $\left(u_{i}, v_{1}\right)$ be colored $c_{p}$, and color $\left(u_{i}, v_{2}\right)$ with $c_{b} \in L^{\prime}\left(u_{i}, v_{2}\right)$ such that $c_{b} \neq c_{p}$. Now $L^{\prime}$-list-color the rest of the vertices.

We claim that in each of the three cases above the coloring is list-distinguishing. First consider an automorphism of the form $\left(\sigma_{n}^{a} \tau_{n}^{b}, e\right), \sigma_{n}^{a} \tau_{n}^{b} \neq e$, which maps $\left(u_{t}, v_{1}\right)$ to $\left(u_{r}, v_{1}\right)$ for some $t \neq r$ by Lemma 7 . The graph induced by the vertices of $C_{n}^{1}$ has been assigned a distinguishing coloring. Thus an automorphism of this form does not preserve colors.

Also by Lemma 7 , the automorphism $\left(e, \sigma_{m}^{c} \tau_{m}^{d}\right), \sigma_{m}^{c} \tau_{m}^{d} \neq e$, maps either $\left(u_{i}, v_{2}\right)$ to $\left(u_{i}, v_{j}\right), 2 \neq j$ for all $i \in\{1, \ldots, n\}$ or $\left(u_{1}, v_{1}\right)$ to $\left(u_{1}, v_{j}\right), 1 \neq j$. However in case $1, n-1$ vertices of $C_{n}^{2}$ are colored $c_{1}$, and this is the only fiber with such a coloring. The vertex $\left(u_{1}, v_{1}\right)$ is colored $c_{1}$ while the rest of the fiber $C_{n}^{1}$ is not; again this is the only fiber with such a coloring. In Case $2,\left(u_{1}, v_{1}\right)$ is the only vertex colored $c_{1}$, and we know that $\left(u_{i}, v_{2}\right)$ must map to $\left(u_{i}, v_{m}\right)$, which are colored differently. In Case $3,\left(u_{1}, v_{1}\right)$ and $\left(u_{1}, v_{2}\right)$ are the only two vertices colored $c_{1}$, so any non-trivial automorphism must interchange $\left(u_{1}, v_{1}\right)$ and $\left(u_{1}, v_{2}\right)$, which will also interchange $\left(u_{i}, v_{1}\right)$ and $\left(u_{i}, v_{2}\right)$, which have different colors. Therefore in each of these cases, automorphisms of this type are not color-preserving. 
The automorphism $\left(\sigma_{n}^{a} \tau_{n}^{b}, \sigma_{m}^{c} \tau_{m}^{d}\right)$ is equal to $\left(e, \sigma_{m}^{c} \tau_{m}^{d}\right) \circ\left(\sigma_{n}^{a} \tau_{n}^{b}, e\right)$. In Case 1 , the automorphism $\left(\sigma_{n}^{a} \tau_{n}^{b}, e\right)$ will map $\left(u_{1}, v_{1}\right)$ to $\left(u_{j}, v_{1}\right)$ for some $j \in\{1 \ldots n\}$ and $\left(u_{i}, v_{2}\right)$ to $\left(u_{k}, v_{2}\right)$ for all $i \in\{1, \ldots, n\}$. But $n-1$ vertices of $C_{n}^{2}$ are colored with $c_{1}$ and the vertex $\left(u_{1}, v_{1}\right)$ is in fiber $C_{n}^{1}$, so we are in the previous case when performing $\left(\sigma_{n}^{a} \tau_{n}^{b}, e\right)$. Cases 2 and 3 will similarly result in the previous case when performing $\left(\sigma_{n}^{a} \tau_{n}^{b}, e\right)$. Therefore in each of these cases, automorphisms of this type are not color-preserving.

If $n=m$ consider the automorphism $\alpha$, such that $\alpha\left(u_{i}, v_{1}\right) \mapsto\left(u_{1}, v_{j}\right)$ for some $i$ and $j$, which would map $C_{m}^{1}$ to $C_{n}^{1}$. In Case $1, C_{n}^{2}$ has $n-1$ vertices colored $c_{1}$ and there is no $C_{m}$ fiber with this number of vertices colored $c_{1}$. In Case 2, we have broken $\alpha$ by coloring $\left(u_{i}, v_{1}\right)$ and $\left(u_{1}, v_{j}\right)$ differently. In Case $3, C_{m}^{1}$ has two vertices colored $c_{1}$ and no $C_{n}$ fiber has more than one vertex colored $c_{1}$. Therefore in each of these cases, automorphisms of this type are not color-preserving.

In every case, colors can be chosen from the list of size 2 to list-distinguish $C_{n} \square C_{m}$. If there exists a fiber that can be $L$-list-distinguished, $|L(v)|=2$ for all $v \in V(G)$, with a vertex that has a unique color for that fiber, then $G$ can be $L$-list-distinguished.

In Figure $4, \sim c$ refers to any color that is not $c$.

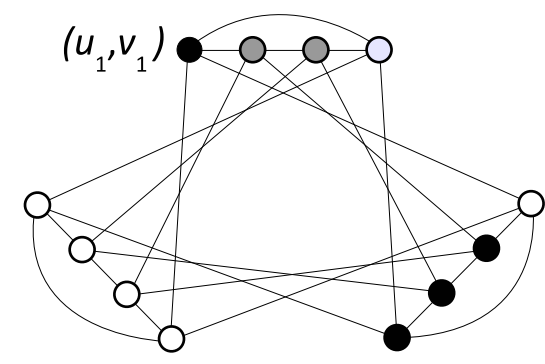

Case 1

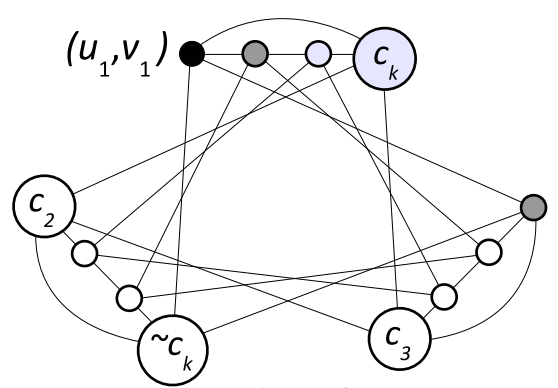

Case 2

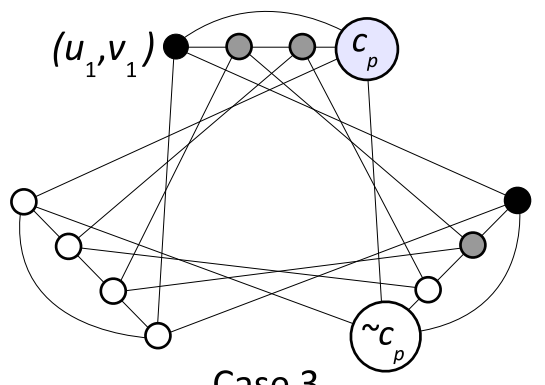

Case 3

Figure 4: Examples of colorings described in Lemma 8. Vertices represented by open circles cannot be colored black.

Lemma 9. Let $n \geq 3$ and $m \geq 3$ such that at most one of $n$ and $m$ is 3 . Then $D_{\ell}\left(C_{n} \square C_{m}\right)=2$. 
Proof. Assign a list $L\left(u_{i}, v_{j}\right)$ such that $\left|L\left(u_{i}, v_{j}\right)\right|=2$ to each $\left(u_{i}, v_{j}\right) \in V\left(C_{n} \square C_{m}\right)$. If $\left|\cup L\left(u_{i}, v_{j}\right)\right|=2$, then color the vertices as in [BC04] if $n=m=4$ or as in [IK06] otherwise. If not, then there must be two vertices whose lists are different. Since $C_{n} \square C_{m}$ is connected, there exist two adjacent vertices with different lists, and these two vertices are either on the same $C_{n}$ or $C_{m}$ fiber. Without loss of generality, assume they are the vertices $\left(u_{1}, v_{1}\right)$ and $\left(u_{2}, v_{1}\right)$ on $C_{n}^{1}$. Color the vertex $\left(u_{n}, v_{1}\right)$ with $c_{3} \in L\left(u_{n}, v_{1}\right)$. Since their lists are not the same, color vertices $\left(u_{1}, v_{1}\right)$ and $\left(u_{2}, v_{1}\right)$ from their list such that the colors are different and both not $c_{3}$, say $c_{1}$ and $c_{2}$ respectively. Color the rest of the vertices of $C_{n}^{1}$ anything from their list other than $c_{1}$.

Since $\left(u_{1}, v_{1}\right)$ is the only vertex with color $c_{1}$, any color-preserving automorphism on the fiber $C_{n}^{1}$ must map $\left(u_{1}, v_{1}\right)$ to itself. Therefore, either $\left(u_{n}, v_{1}\right) \mapsto\left(u_{2}, v_{1}\right)$ or $\left(u_{n}, v_{1}\right) \mapsto$ $\left(u_{n}, v_{1}\right)$. In the former case, the automorphism does not preserve the colors. This leaves us with only the latter, which is the trivial automorphism, so this is an $L$-list-distinguishing coloring on $C_{n}^{1}$ that uses a unique color $c_{1}$. By Lemma 8, we know that we can extend this coloring to a $L$-list-distinguishing coloring of $C_{n} \square C_{m}$. Therefore, $D_{\ell}\left(C_{n} \square C_{m}\right)=2$ if at most one of $n$ and $m$ is 3 .

Lemma 10. $D_{\ell}\left(C_{3} \square C_{3}\right)=D\left(C_{3} \square C_{3}\right)=3$.

Proof. Assign list $L\left(u_{i}, v_{j}\right)$ such that $\left|L\left(u_{i}, v_{j}\right)\right|=3$ to each $u_{i}, v_{j} \in V\left(C_{3} \square C_{3}\right)$. Color the vertices $\left(u_{i}, v_{1}\right), i \in\{1,2,3\}$ from their list such that each vertex received a unique color. Let vertex $\left(u_{i}, v_{1}\right)$ for $i \in\{1,2,3\}$ be colored $c_{i}$, and we will not use the color $c_{1}$ on any other vertex. If one of the vertices $\left(u_{1}, v_{2}\right)$ and $\left(u_{1}, v_{3}\right)$ has a color that is not $c_{1}, c_{2}$, or $c_{3}$ in its list, say $c_{4}$, then use that color. Then color the other anything from its list that is not $c_{1}$. If both of their lists are $\left\{c_{1}, c_{2}, c_{3}\right\}$, then color them both $c_{2}$. Lastly, color $\left(u_{3}, v_{2}\right)$ and $\left(u_{3}, v_{3}\right)$ from their list such that the colors are different and both not $c_{1}$, and then color the remaining vertices anything from their list that is not $c_{1}$.

By Lemma 7 and since $C_{3}^{1}$ has a list-distinguishing coloring, there is no color-preserving automorphism of the form $\left(\sigma_{n}^{a} \tau_{n}^{b}, e\right)$. Since $\left(u_{1}, v_{1}\right)$ is the only vertex with color $c_{1}$, the only non-trivial automorphism of the form $\left(e, \sigma_{m}^{c} \tau_{m}^{d}\right)$ or $\left(\sigma_{n}^{a} \tau_{n}^{b}, \sigma_{m}^{c} \tau_{m}^{d}\right)$ would map $\left(u_{3}, v_{2}\right)$ to $\left(u_{3}, v_{3}\right)$. However, these vertices are colored differently. Lastly, the automorphism $\alpha:\left(u_{i}, v_{j}\right) \mapsto\left(v_{j}, u_{i}\right)$ must map $\left(u_{1}, v_{1}\right)$ to itself. However, $\left(u_{2}, v_{1}\right)$ and $\left(u_{3}, v_{1}\right)$ are colored differently than $\left(u_{1}, v_{2}\right)$ and $\left(u_{1}, v_{3}\right)$. Thus the only color preserving automorphism is the identity, and $D_{\ell}\left(C_{3} \square C_{3}\right)=D\left(C_{3} \square C_{3}\right)=3$.

The proof of the next theorem follows immediately from Lemma 9 and Lemma 10.

Theorem 4. $D_{\ell}\left(C_{n} \square C_{m}\right)=D\left(C_{n} \square C_{m}\right)$ for all $n, m \geq 3$.

Klavžar and Zhu [KZ07] and Fischer and Isaak [FI08] independently determined the distinguishing number of Cartesian products of complete graphs. Again, list-distinguishing this class of graphs has proved more difficult. For instance, a tedious (yet straightforward) case analysis yields $D_{\ell}\left(K_{3} \square K_{4}\right)=2$. As we feel that the development of more broadly 
applicable techniques may increase the overall understanding of the list-distinguishing number, we pose the following problem.

Problem 1. Determine $D_{\ell}\left(K_{n} \square K_{m}\right)$.

\section{Conclusion}

When presenting a list variant $p_{\ell}$ of a graph parameter $p$, it is natural to ask whether $p$ and $p_{\ell}$ differ significantly. For instance, it is well known that the list chromatic number and the chromatic number can differ by an arbitrarily large amount [Alo93, ERT80]. On the other hand, the list coloring conjecture states that the edge chromatic number and edge-list-chromatic number are always equal and remains one of the foremost open problems in chromatic graph theory. With this in mind, we pose the following question.

Question 1. Does there exist a graph $G$ such that $D(G)<D_{\ell}(G)$ ?

Amusingly, the first author feels that no such graph $G$ exists, while the third author believes that Question 1 can be answered in the affirmative. As such, we make no formal conjecture related to Question 1 here.

Acknowledgment: The authors would like to thank Dan Cranston and Stephen Hartke for their input on this paper, and in particular for their part in Proposition 1, which was proved jointly with the first author during a discussion at the 2008 SIAM Discrete Mathematics Meeting.

\section{References}

[AC96] Michael O. Albertson and Karen L. Collins. Symmetry breaking in graphs. Electron. J. Combin., 3(1):Research Paper 18, approx. 17 pp. (electronic), 1996.

[Alb05] Michael O. Albertson. Distinguishing Cartesian powers of graphs. Electron. J. Combin., 12:Note 17, 5 pp. (electronic), 2005.

[Alo93] Noga Alon. Restricted colorings of graphs. In Surveys in Combinatorics 1993, London Math. Soc. Lecture Notes Series 18\%, pages 1-33. Cambridge University Press, 1993.

[BC04] Bill Bogstad and Lenore J. Cowen. The distinguishing number of the hypercube. Discrete Math., 283(1-3):29-35, 2004.

[Bou09] Debra L. Boutin. The determining number of a Cartesian product. J. Graph Theory, 61(2):77-87, 2009.

[CHK10] Jeong Ok Choi, Stephen G. Hartke, and Hemanshu Kaul. Distinguishing chromatic number of Cartesian products of graphs. SIAM J. Discrete Math., 24(1):82-100, 2010. 
[CT06] Karen L. Collins and Ann N. Trenk. The distinguishing chromatic number. Electron. J. Combin., 13(1):Research Paper 16, 19 pp. (electronic), 2006.

[ERT80] Paul Erdős, Arthur Rubin, and Herbert Taylor. Choosability in graphs. Congr. Numer., 26:125-157, 1980.

[FI08] Michael Fischer and Garth Isaak. Distinguishing colorings of cartesian products of complete graphs. Discrete Math, 308:2240-2246, 2008.

[IK06] Wilfried Imrich and Sandi Klavžar. Distinguishing Cartesian powers of graphs. J. Graph Theory, 53(3):250-260, 2006.

[Imr67] W. Imrich. Kartesisches Produkt von Mengensystemen und Graphen. Studia Sci. Math. Hungar., 2:285-290, 1967.

[KWZ06] Sandi Klavžar, Tsai-Lien Wong, and Xuding Zhu. Distinguishing labellings of group action on vector spaces and graphs. J. Algebra, 303(2):626-641, 2006.

[KZ07] Sandi Klavžar and Xuding Zhu. Cartesian powers of graphs can be distinguished by two labels. European J. Combin., 28(1):303-310, 2007.

[RS98] Alexander Russell and Ravi Sundaram. A note on the asymptotics and computational complexity of graph distinguishability. Electron. J. Combin., 5:Research Paper 23, 7 pp. (electronic), 1998.

[Wes01] Douglas B. West. Introduction to graph theory. Prentice Hall Inc., Upper Saddle River, NJ, second edition, 2001. 